\title{
THE GENERALIZED SAMPLING THEOREM FOR TRANSFORMS OF NOT NECESSARILY SQUARE INTEGRABLE FUNCTIONS
}

\author{
A. J. JERRI \\ Department of Mathematics and Computer Science \\ Clarkson College of Technology \\ Potsdam, N.Y. 13676 U.S.A.
}

(Received February 1, 1982)

ABSTRACT. It is known that the generalized sampling theorem is valid for certain finite limit integral transforms of square integrable functions. In this note, we will extend the validity of the theorem to include transforms of absolutely integrable functions associated with differentiable kernels. In the proof, we will use the Hölder inequality and a known theorem concerning the uniform convergence of the orthogonal series to the differentiable kernel of the particular integral transform. KEY WOROS AND PHRASES. Sampling theorem, integral transforms, absolutely integrable functions.

1980 MATHEMATICS SUBJECT CLASSIFICATION CODE. Primary 44A10, 41 A05, 93057.

\section{INTRODUCTION}

In a previous paper [1], we presented various methods for proving the Shannon sampling expansion of band limited functions, to include absolutely integrable Fourier transforms besides the usual square integrable ones. In this note, we will use the Hölder inequality to extend the generalized sampling theorem [2] to finite limit integral transforms of absolutely integrable functions. An example is the following finite limit $J_{0}$-Hankel (Bessel) transform

$$
\frac{\sin a t}{t}=\int_{0}^{a} \frac{J_{0}(x t) d x}{\sqrt{a^{2}-x^{2}}}
$$

of the function $F(x)=\frac{1}{x \sqrt{a^{2}-x^{2}}}$ which is absolutely integrable but not square integrable on the interval $(0, a)$ with respect to the weighting function $\rho(x)=x$. Before we state the generalized sampling theorem and give the proof for its present relaxed version, we will introduce a few basic definitions and the Hölder inequality [3]. $f(x) \& L^{p}(a, b)$ means that $f(x)$ is Lebesque measurable and that $\int_{a}^{b}|f(x)|^{p} d x<\infty$. 
The norm $\|f\|_{p}$ of $f$ is defined by

$$
\|f\|_{p}=\left[\int_{a}^{b}|f(x)|^{p} d x\right]^{\frac{1}{p}} \text {. }
$$

The following inclusion relation may prove valuable. For $k \geq 0$ and $0<\mathrm{q}<\mathrm{p}<\infty$

$$
C^{\infty} \subset \ldots \subset C^{k+1} \subset C^{k} \subset \ldots \subset C^{0} \subset L^{\infty} \subset L^{p} \subset L^{q}
$$

where $C^{k}$ stands for the set of functions which are k-times continuously differentiable. A sequence $S_{N}(x)$ is said to converge in the mean of order $p$ to $f(x)$ if

$$
\lim _{N \rightarrow \infty} \int_{a}^{b}\left|f-S_{N}(x)\right|^{p} d x=0 .
$$

For $p=\infty$, this convergence is equivalent to uniform convergence in the sense of the norm $\|f\|_{\infty}[3]$.

HÖLDER INEQUALITY: For a finite or infinite interval, let $f \varepsilon L^{p}$ and $g \varepsilon L^{p^{\prime}}$, where $1 \leq \mathrm{p} \leq \infty$ and $\frac{1}{\mathrm{p}}+\frac{1}{\mathrm{p}^{\prime}}=1$; then

$$
\int|f g| d x \leq\left[\int|f|^{p} d x\right]^{\frac{1}{p}}\left[\int|g|^{p^{\prime}} d x\right]^{\frac{1}{p^{\prime}}} .
$$

\section{THE GENERALIZED SAMPLING THIEOREM}

The statment of the generalized sampling theorem is: Let I be an interval. Suppose that for each real $t$

$$
f(t)=\int_{I} K(t, x) g(x) d x
$$

where $g(x) \in L^{2}(I), K(t, x) \varepsilon L^{2}(I)$, and $\left\{K\left(t_{n}, x\right)\right\}$ is a complete orthogonal set on $L^{2}(I)$. Then

$$
f(t)=\lim _{N \rightarrow \infty}|n|^{\sum} \leq N\left(t_{n}\right) S_{n}(t)
$$

where

$$
S_{n}(t)=\frac{\int_{I} K(t, x) \overline{K\left(t_{n}, x\right)} d x}{\int_{I} K\left(t_{n}, x\right)^{2} d x} .
$$

Unless otherwise indicated, summations like $\Sigma c_{n}$ will assume summation limits as in (2.2). Shannon's sampling theorem represents a special case of this theorem corresponding to $k(t, x)=e^{i x t}$ on the interval $[-a, a]$. The origin and various extensions and applications of the sampling theorems are discussed in detail in a tutorial review article by the author [4] with nearly 250 references. In this article, we only hint at the possibility, among other remarks, of the following extension. Here we present the relaxed version of this generalized sampling theorem to 
allow $g(x) \varepsilon L^{p}(a, b)$ with $1 \leq p \leq 2$ instead of $g(x) \varepsilon L^{2}(a, b)$ only, for all differentiable kernels $K(-, x)$.

THEOREM. The above generalized sampling expansion (2.1)-(2.3) is valid for $g(x) \varepsilon L^{p}(a, b)$ where $1 \leq p \leq 2$, for all differentiable kernels $K(-, x)$.

The proof will rely mainly on using the Hölder inequality (1.5) instead of its special case, the Schwarz inequality $\left(p^{\prime} p^{\prime}=2\right)$, which was used in proving the above generalized sampling theorem by Kramer [2]. Consider $S_{N}(t)$ and $D_{N}(x)$, the partial sums of the sampling and orthogonal expansions for $f(t)$ and $K(t, x)$ respectively:

$$
\begin{gathered}
S_{N}(t)=\sum_{n=1}^{N} f\left(t_{n}\right) S_{n}(t) \\
D_{N}(x)=\sum_{n=1}^{N} c_{n} K\left(t_{n}, x\right)=\sum_{n=1}^{N} S_{n}(t) K\left(t_{n}, x\right) .
\end{gathered}
$$

We note that the sampling function $S_{n}(t)$ in (2.3) is the Fourier coefficient of the kernel $K(t, x)$ in terms of the complete orthogonal set $\left\{K\left(t_{n}, x\right)\right\}$.

$$
\begin{gathered}
\left|f(t)-S_{N}(t)\right|=\left|f(t)-\Sigma f\left(t_{n}\right) S_{N}(t)\right| \\
=\int_{I}\left[K(t, x)-\Sigma K\left(t_{n}, x\right) S_{n}(t)\right] g(x) d x \mid \leq\left[\int_{I} \mid K(t, x)-\right. \\
\left.\left.\Sigma K\left(t_{n}, x\right) S_{n}(t)\right|^{p} d x\right]^{\frac{1}{p}}\left[\int_{I}|g(x)|^{p^{\prime}} d x\right]^{\frac{1}{p^{\prime}}}
\end{gathered}
$$

after using equations (2.1) for $f(t),(2.4)$ for $s_{N}(t),(2.1)$ for $f\left(t_{n}\right)$ in $(2.4)$, and the Hölder inequality (1.5). The convergence in (2.6) depends on the type of $L^{p}$ convergence of $\mathrm{D}_{\mathrm{N}}(\mathrm{x})$ to the kernel $\mathrm{K}(-, \mathrm{x})$, which in turn dictates the condition $g(x) \in L^{p^{\prime}}(a, b)$ with $\frac{1}{p}+\frac{1}{p^{\prime}}=1$. But since the orthogonal expansion (2.5) for $K(-, x)$ is done in $L^{2}(a, b)$, we are left with $2 \leq p \leq \infty$ and so $1 \leq p^{\prime} \leq 2$. As we mentioned earlier, the convergence in $L^{\infty}$ sense is equivalent to uniform convergence. Hence, it remains to show that $\mathrm{D}_{\mathrm{N}}(\mathrm{x})$ in (2.5) converges uniformly to the kernel $\mathrm{K}(-, \mathrm{x})$. We first note that $D_{N}(x)$ is an orthogonal expansion of the kernel $K(t, x)$ in terms of its discrete values $K\left(t_{n}, x\right)$, which for all practical purposes are taken to be the solutions of the nth order self-adjoint Sturm-Liouville eigenvalue problem [5]. Uniform convergence is assured (Edwards [3, p. 197 ) for such orthogonal expansions to differentiable functions that also satisfy the boundary conditions of the SturmLiouville problem. The latter boundary condition on the particular function in question $K(t, x)$ is clearly satisfied as it determines $t_{n}$ for $K\left(t_{n}, x\right)$.

The convergence of the generalized sampling series of the example in (1.1) is 
now assured since $J_{0}(x t)$ is differentiable on $(0,1)$.

3. CONCLUSIONS

The importance of the generalized sampling theorem and hence its present relaxed version lies in its use in system analysis. For example, the Hankel transform [6] is used to replace the double Fourier transform in treating problems with circular symmetry, particularly in optics. The generalized sampling theorem was also used for the analysis of time-varying systems [7] and the recent development of discrete Hankel (Bessel) and classical orthogonal polynomial transforms $[8,9]$.

\section{REFERENCES}

1. JERRI, A.J., Sampling for Not Necessarily Finite Energy Signals, Int. J. System Sci. , $\underline{4}, 255-260$ (1973).

2. KRAMER, H.P., A Generalized Sampling Theorem, J. Math. Phys., 38, 68-72 (1959).

3. EDWARDS, R.E., Fourier Series, A Modern Introduction, Vol. 1,2, Holt, Rinehart and Wiston, New York, 1967.

4. JERRI, A.J., The Shannon Sampling Theorem - Its Various Extensions and Applications: A Tutorial Review, Proc. IEEE, 65, 1565-1596 (1977).

5. CODDINGTON, E.A. and LEVINSON, N., The Theory of Ordinary Differential Equations, McGraw-Hil1, New York, 1955.

6. PAPOULIS, A., Systems and Transforms with Applications in Optics, McGraw-Hill, New York, 1968 .

7. JERRI, A.J., Application of the Sampling Theorem to Time-Varying Systems, J. Franklin Inst., 293, 53-58 (1972).

8. JERRI, A.J., Towards a Discrete Hankel Transform, Applicable Anal., 7, 97-109 (1978).

9. JERRI, A.J., The Application of General Discrete Transforms to Computing Orthogonal Series and Solving Boundary Value Problems, "Bull. Calcutta Math Soc., 71, 177-187, (1979). 


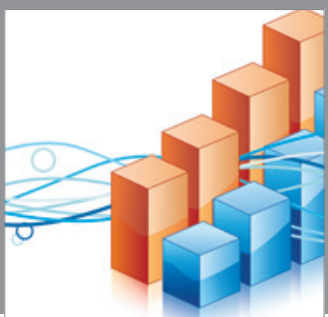

Advances in

Operations Research

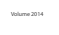

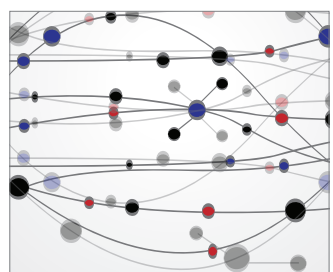

\section{The Scientific} World Journal
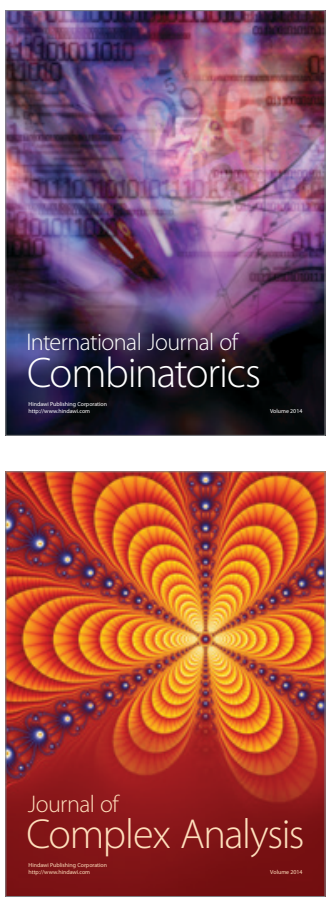

International Journal of

Mathematics and

Mathematical

Sciences
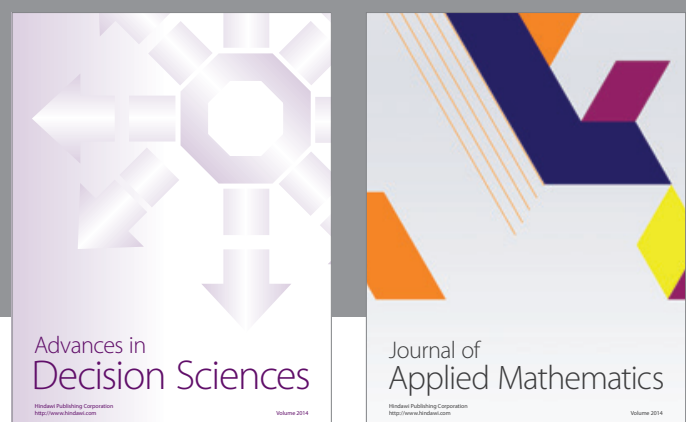

Journal of

Applied Mathematics
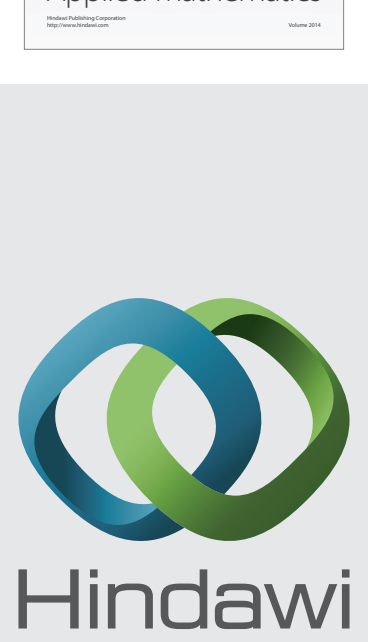

Submit your manuscripts at http://www.hindawi.com
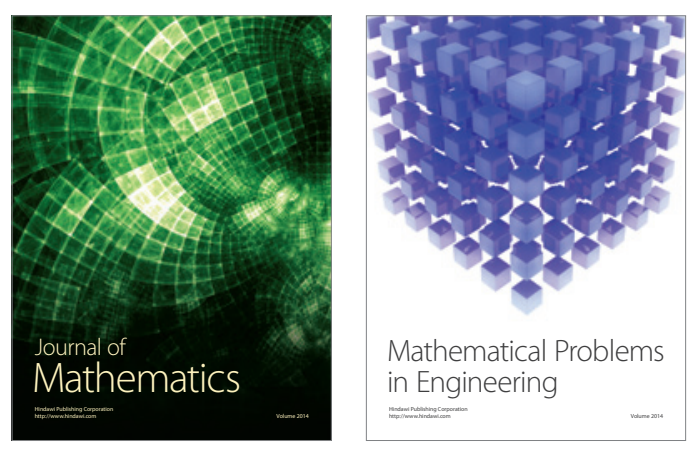

Mathematical Problems in Engineering
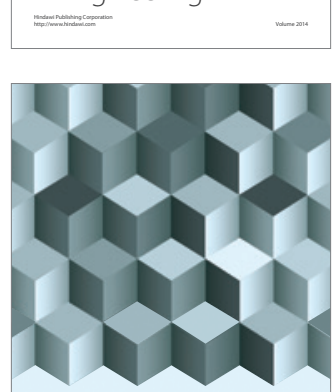

Journal of

Function Spaces
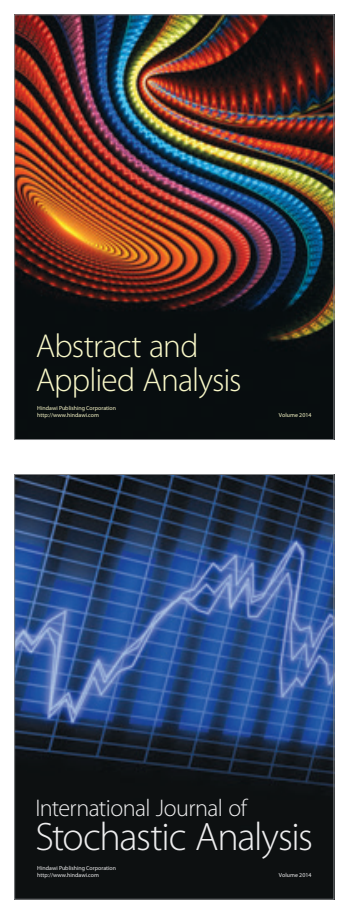

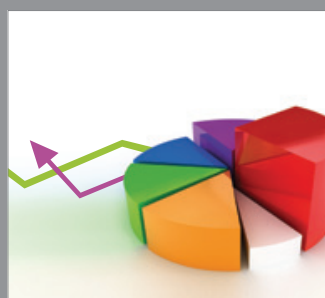

ournal of

Probability and Statistics

Promensencen
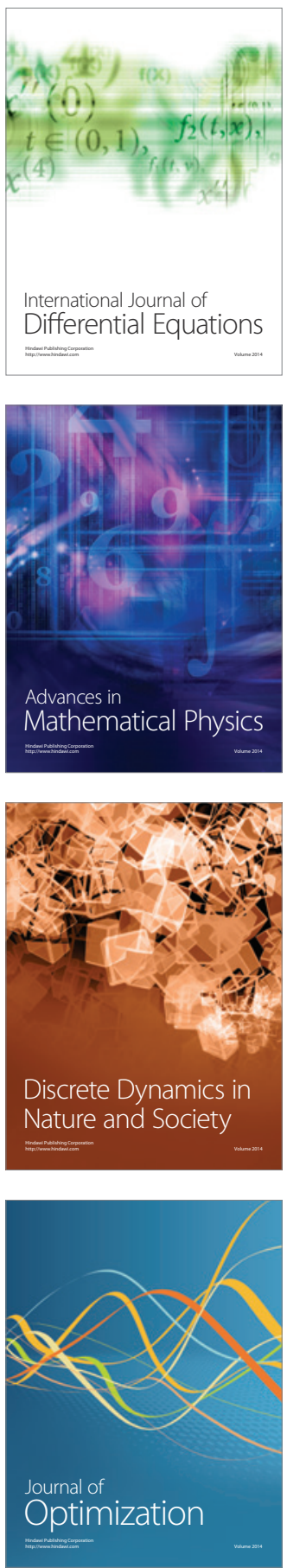